\title{
Pure Motor Monoparesis in the Upper Extremity Due to a Medial Medullary Infarction
}

\author{
Hiromasa Tsuda ${ }^{a}$,, Naoya Otsubo ${ }^{\text {a }}$, Masaki Ishihara ${ }^{\mathrm{a}}$
}

\begin{abstract}
A 69-year-old man with essential hypertension abruptly presented with moderate pure motor monoparesis (PMM) in the left-sided upper extremity. There were no other neurologic abnormalities. Cranial magnetic resonance imaging demonstrated a small infarction in the caudal and lateral region of the right-sided medial medulla oblongata. Thereafter, intravenous anti-thrombin agent was initiated, and she became asymptomatic within 14 days. This is a first reported case of PMM in the upper extremity due to medial medullary infarction (MMI). In the medulla oblongata, we speculated that the corticospinal tract fibers innervating the lower extremity might decussate rostral to the fibers innervating the upper extremity.
\end{abstract}

Keywords: Corticospinal tract; Dejerine syndrome; Medial medullary syndrome; Medulla oblongata; Pyramidal decussation; Pyramidal tract

\section{Introduction}

In medial medullary infarction (MMI), various neurological symptoms, such as hemiparesis, hypoglossal nerve palsy and sensory disturbance commonly occur [1]. On the other hand, pure motor monoparesis (PMM) in the upper extremity is characterized by weakness limited to unilateral upper limb without other neurological abnormalities, and caused by isolated involvement of the corticospinal tract [2]. Regarding responsible infarct lesions for PMM in the upper extremity, the cerebral cortex, corona radiata, internal capsule and pons have been

Manuscript accepted for publication November 13, 2014

${ }^{\text {a }}$ Department of Neurology, Tokyo Metropolitan Health and Medical Corporation Toshima Hospital, Japan

${ }^{\mathrm{b}}$ Corresponding Author: Hiromasa Tsuda, Department of Neurology, Tokyo Metropolitan Health and Medical Corporation Toshima Hospital, 33-1, Sakaecho, Itabashi-ku, 173-0015 Tokyo, Japan.

Email: hiromasa_tsuda@tokyo-hmt.jp

doi: http://dx.doi.org/10.14740/jmc1989w noted [2]. Here, we describe a first reported case of PMM in the upper extremity due to an MMI. Moreover, this case may be useful for elucidate the topographical organization of the corticospinal tract fibers in the medulla oblongata.

\section{Case Report}

A 69-year-old man with essential hypertension complained of abruptly presenting with difficulty in lifting the left arm, despite no shoulder pain, and was admitted to our Neurologic Ward on July 2014. Consciousness was alert. Blood pressure was $154 / 84 \mathrm{~mm} \mathrm{Hg}$. The heart rate was $62 / \mathrm{min}$. General examination demonstrated no abnormalities. Cranial nerve impairment was not detected. The patient was right-handed. Hand grasping power was $31 \mathrm{~kg}$ in the right and $29 \mathrm{~kg}$ in the left. In the upper extremities, left-sided Barre sign was positive. In the lower extremities, neither Barre sign nor Mingazzini sign was positive. Manual muscle testing on the left upper extremity demonstrated that pectoralis major, deltoid, supraspinatus, infraspinatus, rhomboideus, serratus anterior, latissimus dorsi, trapezius, biceps brachii, triceps brachialis, and brachioradialis were at grade 4 , while extensor carpi radialis longus, extensor carpi ulnaris, flexor carpi radialis, and flexor carpi ulnaris at grade 5-. Muscle tonus was normal in the extremities. However, manual muscle testing on the right upper extremity and bilateral lower extremities demonstrated no abnormalities. Muscle atrophy and fasciculation were not observed. Deep tendon reflexes were all normal and pathological reflexes were not detected in the extremities. Superficial sensation (touch sensation, pain sensation, temperature sensation, and topesthesia), deep sensation (joint sensation and vibratory sense), and combined sensation (two-point discrimination, graphesthesia, stereognosis, and double simultaneous stimulation) were all normal. Dysmetria, decomposition of movement, and intension tremor were not observed in finger-nose test and nose-finger-nose test. Hyperpronation test and hand pronation supination test were slow on the left side. Truncal ataxia was not observed. In the gait test, arm swing was slow on the left side. There were no other neurologic abnormalities. As a result, there was moderate weakness in the left upper extremity with proximal dominancy. Complete blood cell count and blood chemistry were within normal ranges. Electrocardiogram, echocardiography, and chest roentgenogram findings 


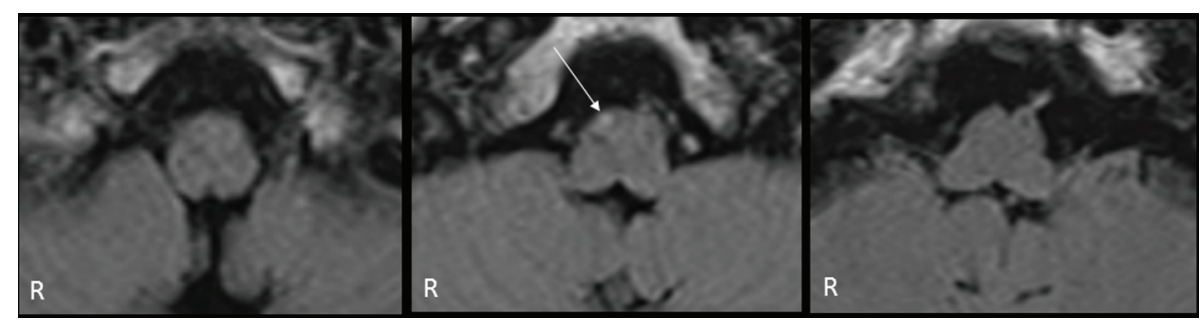

Figure 1. Fluid-attenuated inversion recovery image of cranial magnetic resonance imaging on the axial image demonstrated a small infarct lesion in the medial medulla oblongata on the right side (arrow).

demonstrated no abnormalities. Cranial magnetic resonance imaging demonstrated a localized MMI on the right side (Fig. 1). Cranial magnetic resonance angiography demonstrated no abnormalities. Seven days of intravenous argatroban therapy were performed. Thereafter, oral clopidogrel at $75 \mathrm{mg}$ /day was initiated. Under rehabilitation, the patient became asymptomatic within 14 days.

\section{Discussion}

Although the somatotopic arrangements of the corticospinal tract in the human brain have been generally elucidated [3], very little is known about the somatotopy at the medullary pyramid. In 2011, based on the diffusion tensor tractography analysis, Kwon et al [4] reported that the hand somatotopy of the corticospinal tract was located at the medial portion of the medullary pyramid; in contrast, the leg somatotopy occupied its lateral portion. However, in our patient, despite PMM in the upper extremity, a localized infarct lesion was detected in the lateral portion of the medial medulla oblongata. This neurological symptom was incompatible with the diffusion tensor tractography analysis which was reported by Kwon et al [4].

There were only two reported cases of lateral medullary infarction with ipsilateral monoparesis of the leg $[5,6]$. In both cases, infarct lesion was observed in the most caudal region of lateral medulla oblongata. Regarding the etiology of leg weakness due to ipsilateral lateral medullary infarction, Liu et al [5] and Tsuda et al [6] speculated that the corticospinal tract fibers innervating the lower and upper extremities might decussate at different levels, and that the decussation of the lower extremity might locate rostral to that of the upper extremity. In our patient, an infarct lesion was located in the caudal region in the medial medulla oblongata. Therefore, we agree with the hypothesis which was proposed by Liu et al [5] and Tsuda et al [6]. In addition, we emphasize that this is a first reported case of PMM in the upper extremity due to an MMI.

\section{Conflict of Interest}

The authors declare no conflict of interest.

\section{Grant Support}

None.

\section{References}

1. Kameda W, Kawanami T, Kurita K, Daimon M, Kayama T, Hosoya T, Kato T. Lateral and medial medullary infarction: a comparative analysis of 214 patients. Stroke. 2004;35(3):694-699.

2. Paciaroni M, Caso V, Milia P, Venti M, Silvestrelli G, Palmerini F, Nardi K, et al. Isolated monoparesis following stroke. J Neurol Neurosurg Psychiatry. 2005;76(6):805807.

3. Jang SH. Somatotopic arrangement and location of the corticospinal tract in the brainstem of the human brain. Yonsei Med J. 2011;52(4):553-557.

4. Kwon HG, Hong JH, Lee MY, Kwon YH, Jang SH. Somatotopic arrangement of the corticospinal tract at the medullary pyramid in the human brain. Eur Neurol. 2011;65(1):46-49.

5. Liu CY, Chang FC, Hu HH, Hsu LC. Ipsilateral crural monoparesis in lateral medullary infarction due to vertebral artery dissection. Eur J Neurol. 2006;13(7):e8-9.

6. Tsuda H, Tanaka K, Kishida S. Pure Motor Monoparesis in the Leg due to a Lateral Medullary Infarction. Case Rep Med. 2012;2012:758482. 